\title{
Intraktable Diarrhoe aufgrund kongenitaler Enterozytendefekte
}

\author{
Alan D. Phillips \\ Centre for Paediatric Gastroenterology, Royal Free Hospital, London, UK
}

\section{Schlüsselwörter \\ Intraktable Diarrhoe · Säuglings- und Kleinkindalter • Enteropathie}

\section{Zusammenfassung}

Hintergrund: Die intraktable Diarrhoe im Säuglingsalter ist ein ernstes Problem, das mit hoher Morbidität und Mortalität einhergeht. Da keine einfachen Heilmittel zur Verfügung stehen, verlangen diese Fälle die engagierte Pflege der Eltern und des medizinischen Personals. Die geringe Zahl an Patienten weist darauf hin, dass es für ein besseres Verständnis dieser Erkrankungen konzertierter internationaler Bemühungen bedarf, um sowohl Ressourcen als auch Patienten zusammenzutragen. Regelmässige Übersichtsarbeiten über Publikationen auf diesem Gebiet sind zur Bekanntmachung bzw. Verbreitung von erzielten Fortschritten und Hypothesen und um diagnostische Kriterien anzusprechen erforderlich. Methoden: Dieser Artikel bietet einen Überblick über die aktuelle und frühere Literatur zum Thema kongenitale Enterozytendefekte. Ergebnisse: Innerhalb dieses Syndroms sind eigenständige Diagnosen möglich und deren diagnostische Kriterien gewinnen allmählich an Akzeptanz. Berichte über diese Fälle werden weltweit veröffentlicht und im klinischen Verlauf zeichnen sich Unterschiede ab, wobei in manchen Fällen eine Besserung des Schweregrads der Symptome auszumachen ist. Mor- bidität und Mortalität sind jedoch nach wie vor hoch. Schlussfolgerungen: Die Bestimmung der diesen Erkrankungen zugrunde liegenden Ursachen liegt immer noch in weiter Ferne. Dank molekulargenetischer Untersuchungen rückt jedoch die Hoffnung, die genetische Grundlage der Erkrankungen feststellen zu können, in den Bereich des Realistischen. Diese Bestimmung der genetischen Grundlage ist prioritär, da dadurch eindeutigere diagnostische Kriterien, eine Klärung der Krankheitszusammenhänge und die Bereitstellung der dringend benötigten pathogenen Informationen ermöglicht werden sollten.

Copyright (c) 2006 Nestec Ltd., Vevey/S. Karger AG, Basel

\section{Einleitung}

Die intraktable Diarrhoe ist nach wie vor ein ernstes pädiatrisches Problem, das mit hoher Morbidität und Mortalität einhergeht. Sie stellt eine extreme Einzelbelastung dar, die jeglicher Behandlung trotzt. Diese Krankheit verlangt nach engagierter Pflege seitens Eltern und medizinischem Personal. Bei dieser speziellen Form der protrahierten, therapieresistenten Diarrhoe, d.h. bei angeborenen Defekten der Enterozyten, manifestiert sich die Erkrankung häufig bei Säuglingen in den ersten Lebenstagen. Die Komplexität des zur Diagnose und Behandlung erforderlichen Gewebe- und Venenzugangs

\section{KARGER}

Fax +4161306 1234

E-Mail karger@karger.ch

www.karger.com
C) 2006 Nestec Ltd. Vevey/S. Karger AG, Basel 0250-9652/06/0641-0015\$23.50/0

Online-Zugriff auf: www.karger.com/and
Alan D. Phillips, PhD

Centre for Paediatric Gastroenterology

Lower 3rd Floor, Royal Free Hospital, Pond Street

London, NW3 2QG (UK)

Tel. +44 207830 2783,Fax +44 207830 2146, E-Mail a.phillips@medsch.ucl.ac.uk 

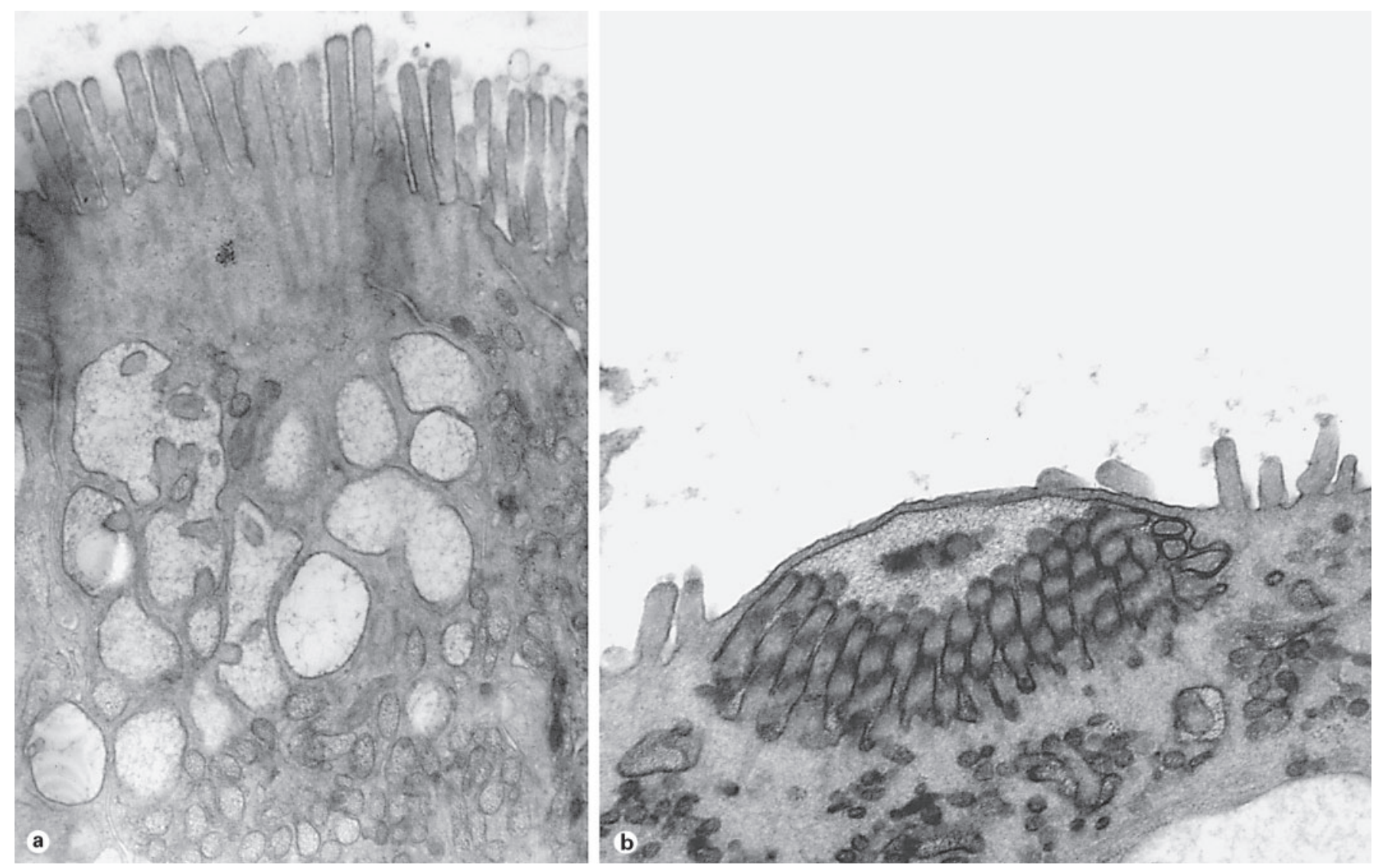

Abb. 1. Mikrovillusatrophie. a Sichtbare Entwicklung der Mikrovilli in einer Zelle mit einem intakten, wenn auch kurzen Bürstensaum. b Blasenartiger Einschluss der Mikrovilli, Anschein von Phagozytose.

verstärkt die Probleme beträchtlich, die sich durch die durchfallbedingten Gewichtsverluste ergeben und die pro Tag bis zu 10\% des Körpergewichts und mehr betragen können. Nichtsdestotrotz sind eigenständige Diagnosen möglich. Dank parenteraler Ernährung ist inzwischen eine relativ lange Überlebensdauer möglich; die Dünndarmtransplantation weckt Hoffnungen auf ein Leben ohne Krankheit und auch die Molekulargenetik kommt bei dem Versuch, die dieser Erkrankung mit Sicherheit zugrunde liegenden genetischen Defekte zu enträtseln, zum Einsatz.

Der Begriff «Intraktable Diarrhoe» wurde in den frühen 60er Jahren des 20. Jahrhunderts von Avery et al. [1] eingeführt. Untersucht wurde eine Gruppe von 20 Kindern unter 3 Monaten mit chronischer Diarrhoe (Dauer über 14 Tage), negativen Stuhlkulturen und Durchfällen, die trotz medizinischer Interventionen persistierten. Diese Kinder mussten alle parenteral ernährt werden und neun der Kinder starben. In den darauffolgenden Jahren wurden ähnliche Berichte veröffentlicht [2, 3], in denen vermutet wurde, dass es sich bei dem Problem um eine Erbkrankheit handelt, die mit Darmschäden einhergeht und wahrscheinlich von mehreren Krankheitsentitäten verursacht wird (familiäre Enteropathie [4] und letale familiäre protrahierte Diarrhoe [5]).

Davidson et al. [4] beschrieben die Enteropathie als Zottenatrophie mit Kryptenhypoplasie ohne Entzündung der Lamina propria, was darauf schliessen lässt, dass sich weder eine Kryptenhyperplasie (wie etwa bei der Zöliakie) noch eine Immunkomponente nachweisen liess. Obwohl spätere Studien die Hypoplasie bei dieser Form der Enteropathie in Frage stellen (siehe unten), ging man dieser Beobachtung nach und untersuchte die Fälle von intraktabler Diarrhoe anhand immunhistologischer Kriterien $[6,7]$. Dies ermöglichte eine Unterscheidung zwischen Immunerkrankungen und Erkrankungen, die vom Epithel ausgehen, so dass die Krankheitskategorien geklärt wurden [7] und eine Optimierung der Behandlungs- 

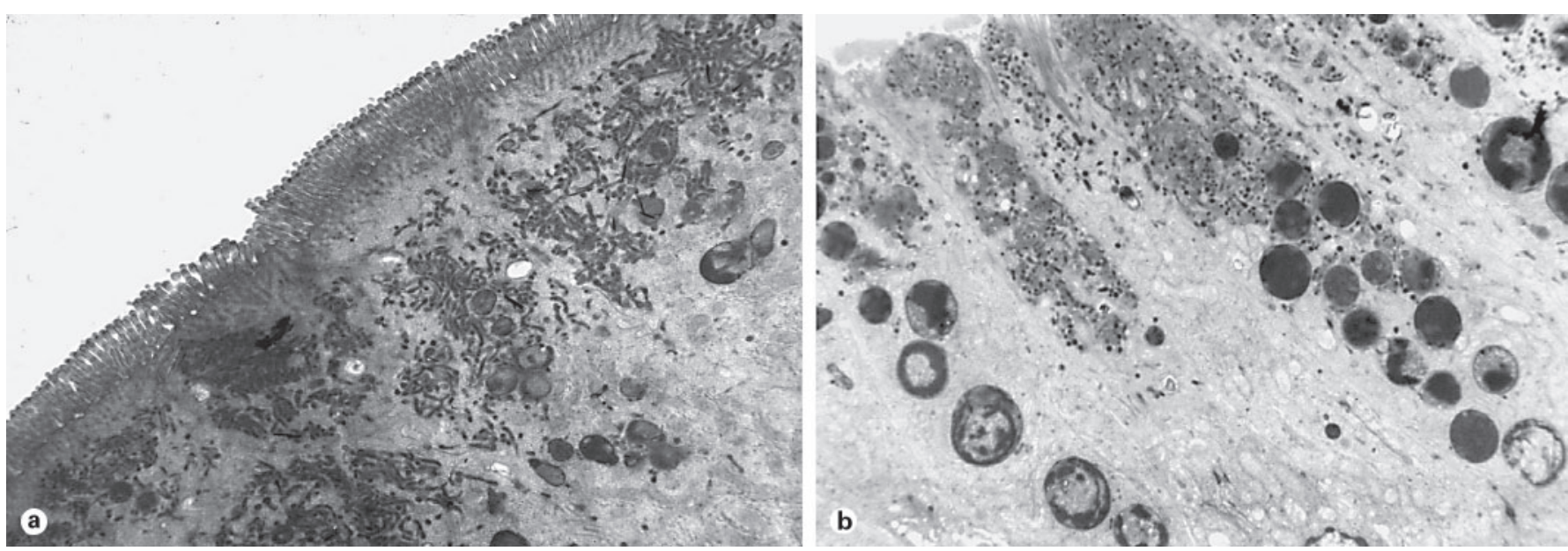

Abb. 2. Mikrovillusatrophie. Sekretgranula bei «late-onset»-Fällen (a) und atypischen Fällen (b).

optionen nahegelegt werden konnte [8]. Dieser Beitrag konzentriert sich auf die vom Epithel ausgehenden Erkrankungen und geht nicht näher auf die AutoimmunEnteropathie bzw. die Immunerkrankungen, die mit dieser verwandt sind, oder die diesbezüglichen therapeutischen Optionen ein, da diese an anderer Stelle behandelt werden.

Ferner wird dieser Beitrag sich auch nicht näher mit der idiopathischen ulzerativen Colitis im Säuglingsalter befassen [9, 19], da deren Merkmale eher einer durch eine Immunerkrankung verursachten intraktablen Diarrhoe ähneln.

In der Kategorie kongenitale Enterozytendefekte in Verbindung mit intraktabler Diarrhoe gelten derzeit einige wenige eigenständige Diagnosen als anerkannt. Der Einteilung in diese Kategorie liegen unterschiedliche Evidenzstufen zugrunde, wobei jedoch allen Fällen eine Enteropathie mit nicht bzw. nur leicht entzündeter Lamina propria gemein ist.

Bisher wurde nicht geklärt, welche Anomalien diesen Erkrankungen zugrunde liegen, und der Nachweis ihrer molekulargenetischen Basis ist gegenwärtig in Bearbeitung. Noch wurden keine Gendefekte entdeckt. $\mathrm{Zu}$ den oben genannten Diagnosen zählen die Mikrovillusatrophie bzw. die Enteropathie mit Einschluss von Mikrovilli, die Tufting-Enteropathie bzw. Epithelzelldysplasie, die syndromatische Diarrhoe bzw. das Tricho-HepatoEnterische Syndrom, die idiopathische frühkindliche Enterocolitis ulcerosa sowie das CDG-Syndrom; es gibt jedoch nichtsdestotrotz nach wie vor auch idiopathische Fälle. Die oben genannten Diagnosen werden nachfol- gend nacheinander besprochen. Zu den häufiger bei intraktabler Diarrhoe gestellten Diagnosen wurden bereits mehrere Übersichtsarbeiten veröffentlicht [7, 9-15], und dieser Beitrag wird sich bemühen, Wiederholungen auf ein geringes Mass zu beschränken.

\section{Microvillusatrophie/Enteropathie mit Einschluss von Mikrovilli}

1982 wurden beim Jahreskongress der ESPGHAN (European Society for Paediatric Gastroenterology, Hepatology and Nutrition) in Madrid zwei Abstracts vorgestellt, welche ungewöhnliche Befunde im Epithel von Säuglingen mit intraktabler Diarrhoe hervorhoben. Schmitz et al. [16] prägten den Begriff Mikrovillusatrophie, wohingegen Goutet et al. [17] sich auf Davidson et al. [4] bezogen und sich der von diesem Team verwendeten, beschreibenden Bezeichnung «familiäre Enteropathie mit hypoplastischer Zottenatrophie» bedienten. Beide Forschergruppen beschrieben einen Einschluss der Mikrovilli im Epithel, ein Befund, der bereits von Davidson et al. [4] bei einer Reihe von 5 Säuglingen beschrieben worden war. Sie berichteten ferner über das Vorliegen von Sekretgranula in den Kryptenzellen und den Enterozyten des Oberflächenepithels [4]. Wir veröffentlichten zwei weitere Fälle von intraktablen Diarrhoe, bei denen jeweils eine Enteropathie mit den gleichen charakteristischen Merkmalen vorlag (d.h. Mikrovillus-Einschluss und Zunahme der Sekretgranula; Abb. 1, 2) und legten den Schluss nahe, dass diese Charakteristika die diagnos- 
tischen Merkmale der Erkrankung sind [18]. Cutz et al. [11] schlugen zu einem späteren Zeitpunkt die Bezeichnung «Mikrovillus-Einschluss-Krankheit» vor, um die Anomalie der Mikrovilli zu betonen.

\section{Klinische Symptome}

Die klinischen Symptome sind gut beschrieben und gelten als schwerwiegend, wobei die Krankheit in der Regel in der ersten Lebenswoche einsetzt $[4,11,12,18]$. Die Diarrhoe persistiert, selbst wenn das Kind nicht enteral ernährt wird und verschlimmert sich bei oraler Ernährung [12]. Die Erkrankung scheint weltweit aufzutreten, und es liegen Berichte über Fälle in Europa [12], dem Mittleren Osten [12], Asien [19, 20] und Nordamerika [11], u.a. auch bei den Indianern Nordamerikas (NavajoReservat Navajo Nation), vor [21]. Aus Afrika liegen nur wenige Berichte vor, obgleich der Verfasser dieses Beitrags die Diagnose Mikrovillusatrophie bei einem afrikanischstämmigen Nordamerikaner bestätigt hat. In Entwicklungsländern kommt der Schweregrad der Erkrankung und die damit verbundene Letalität möglicherweise einer Diagnose zuvor.

In den meisten Fällen manifestieren sich die Symptome in der ersten Lebenswoche, manchmal jedoch treten sie erst nach Ende der Neugeborenenphase ein; diese Fälle werden als «late-onset»-Form der Mikrovillusatrophie bezeichnet [12]. Trotz feiner Unterschiede (siehe unten) sind im Darm die für diese Erkrankung charakteristischen morphologischen Veränderungen festzustellen und begründen die Diagnose. Die Langzeitüberlebenschancen sind bei dieser Erkrankung schlecht, wenngleich bei den «late-onset»-Fällen eine günstigere Prognose für möglich erachtet wird [12]. Noch ist es zu früh, dies als eine definitive Möglichkeit gelten zu lassen, obwohl es sich in einer Fallserie von 23 Patienten bei drei der fünf Überlebenden um «late-onset»-Fälle handelt [12]. Patient Nr. 9 aus dieser Fallserie ist inzwischen 23 Jahre alt und weist bei teilweise parenteraler Ernährung einen relativ guten Zustand auf, wobei jedoch keine Besserung seiner intestinalen Anomalie nachgewiesen werden kann (Ventura A, persönliche Mitteilung).

Da inzwischen mehr Fälle diagnostiziert wurden, wurde auch eine breiteres Spektrum an Symptomen und Behandlungsverläufen beschrieben. Diese sind von daher interessant, da sie möglicherweise Licht auf die zugrunde liegende Pathogenese werfen bzw. als Erinnerung dafür dienen können, diese Diagnose auch in Situationen, die ausserhalb des akzeptierten Dogmas liegen, in Erwägung zu ziehen, wenngleich sich die meisten Fälle wie in der Literatur beschrieben manifestieren $[4,11,12,18]$. In den meisten Fällen besteht kein Zusammenhang mit einem Polyhydramnion, obwohl vor kurzem über einen Fall mit Polyhydramnion und bereits vor der Geburt erkennbarem Darmverschluss berichtet wurde, bei dem die Diagnose dann letztendlich Mikrovillusatrophie lautete [22]. Die Mikrovillusatrophie sollte daher bei möglicher Chloriddiarrhoe [23] bzw. bei möglicherweise unzureichendem Na+/H+-Austausch [24] im Rahmen der Differenzialdiagnose ebenfalls in Betracht gezogen werden. Ein weiterer «late-onset»-Fall (3 Monate) mit charakteristischem Diagnosebefund nahm im Alter von zwei Jahren trotz persistierender Diarrhoe spontan an Gewicht zu und musste im Alter von vier Jahren nicht länger parenteral ernährt werden [25]. In diesem Fall ist der Krankheitsverlauf zwar ungewöhnlich, eine früh durchgeführte Darmtransplantation [26] wäre bei der betroffenen Patientin und möglicherweise auch bei anderen «late-onset»Patienten jedoch nicht angebracht gewesen.

Ein weiterer interessanter Vorfall ergab sich bei einem Patienten, der auf eine Darm- und Lebertransplantation wartete [27]. Nach einer Leberbiopsie traten bei diesem Patienten schwere intraperitoneale Blutungen auf, die einen chirurgischen Eingriff erforderlich machten; es kam zu einem lebensbedrohlichen haemorrhagischen Schock mit Nierenversagen. Gleichzeitig besserte sich die Diarrhoe des Patienten; der Patient hatte eine Woche lang gar keinen Stuhlgang und nach dieser Woche betrug die Stuhlmenge bis zur Transplantation 10-30 ml/kg/Tag. Bei enteraler Ernährung erhöhte sich die Stuhlmenge, eine Veränderung der intestinalen Morphologie war nicht festzustellen. Die Veränderung der sekretorischen Diarrhoe wurde anscheinend durch eine extreme Senkung des hydrostatischen Drucks bewirkt.

Angesichts der Zahl der konsanguinen Eltern und der Tatsache, dass man Familien gefunden hat, bei denen mehrere Geschwister betroffen sind, handelt es sich wahrscheinlich um eine autosomal rezessive Krankheit [12, 28]. Gleichzeitig auftretende genetische Erkrankungen wurden beschrieben $[29,30]$, wobei jedoch unklar ist, ob es sich hierbei um «Contiguous Gene Syndromes» handelt, d. h. um Syndrome, an deren Ausprägung mehrere Gene in dem betroffenen Chromosomenabschnitt beteiligt sind, oder ob diese unabhängig vererbt werden. Die hohe Zahl der Fälle mit Konsanguinität der Eltern öffnet die Tür für molekulargenetische Studien sowie für die Möglichkeit, die genetische Basis der Erkrankung festzustellen.

Klinisch ähnliche Fälle von intraktabler Diarrhoe, bei denen jedoch die diagnostischen Merkmale der Mikrovillusatrophie im Darm nicht nachzuweisen waren, führten 
zu der Behauptung, diese Fälle seien möglicherweise miteinander verwandt und deuteten auf ein Spektrum diverser Mikrovilluserkrankungen hin [31, 32]. Diese Erkrankungen wurden als intestinale Mikrovillusdystrophie bezeichnet [31]. Die charakteristischen diagnostischen Merkmale wurden möglicherweise übersehen [33] und das Ausmass der Ähnlichkeit mit der Mikrovillusatrophie verlangt nach weiteren Studien, u.a. molekulargenetischen Untersuchungen.

\section{Pathologie}

Es liegt eine Enteropathie mit Zottenatrophie, jedoch ohne starke Kryptenhyperplasie, vor, die zu einer dünnen Schleimhaut führt [12]; die Lamina propria ist nicht entzündet. Es handelt sich hierbei nicht um eine hypoplastische Zottenatrophie [4], da die Zahl der Kryptenzellen erhöht ist $[12,34]$ und eine verstärkte Apoptose und Proliferation vorliegt [35]. Die fehlende Hyperplasie der Kryptenzellen ist nicht auf eine eingeschränkte Reaktionsfähigkeit der Krypten zurückzuführen, da sich bei einer Behandlung mit epidermalem Wachstumsfaktor die Proliferationsrate der Darmzellen erhöht, ohne dass sich dadurch jedoch der klinische Zustand verbessert [34]. Das Absterben der Epithelzellen beeinträchtigt die Verdauung und die Resorption und ist eine der Ursachen für die Diarrhoe.

Die PAS (periodic acid-Schiff)-Färbung zeigt eine abnorme Ansammlung von PAS-positivem Material im apikalen Zytoplasma der Epithelzellen. Bei der kongenitalen Mikrozottenatrophie findet sich diese Ansammlung im oberen Kryptenepithel und die tiefer gelegenen Zellen weisen eine normale PAS-Färbung des Bürstensaums auf. Bei «late-onset»-Fällen dagegen tritt diese Ansammlung von PAS-positivem Material im unteren Zottenepithel auf [12]. In einer dritten Variante, die als «atypische» Mikrovillusatrophie bezeichnet wird, tritt die abnorme Ansammlung von PAS-positivem Material im unteren Teil der Krypte auf und in Richtung des Oberflächenepithels zeigt sich eine in gewissem Masse normale PAS-Färbung des Bürstensaums [12]. Der PAS-Befund deutet darauf hin, dass sich ein für den Bürstensaum bestimmtes Material in der Zelle sammelt, statt in die apikale Membran transportiert zu werden. Diese Verteilung entspricht der im Elektronenmikroskop beobachteten Zunahme der «Sekretgranula». Wahrscheinlich sind diese beiden abnormen Befunde auf das gleiche Phänomen zurückzuführen [36]. Die Sekretgranula enthalten somit Material, das sich normalerweise im Bürstensaum befindet. Eine Untersuchung der Sekretgranula im Elektronenmikroskop zeigte, dass diese nicht einzeln, voneinander getrennt vor- lagen, sondern ein feinblasiges Netz bildeten [36]. Der Golgi-Apparat war normal, was auf eine dem Golgi-Apparat nachgeschaltete Blockade der Exozytose schliessen lässt. Die routinemässige PAS-Färbung eröffnet die Möglichkeit einer retrospektiven Diagnose [36].

Im Elektronenmikroskop zeigt sich das andere Kennzeichen der Mikrovillusatrophie, nämlich das Vorliegen von Vakuolen mit Mikrovillussaum bzw. -einschlüssen im apikalen Zytoplasma der Oberflächenepithelien (Abb. 1), d.h. diese treten zu einem späteren Zeitpunkt im Lebenszyklus einer Epithelzelle auf als die Sekretgranula. Diese Vakuolen können in Form von kleinen oder grossen Einschlüssen auftreten und erscheinen gelegentlich als Einstülpungen der apikalen Membran oder als «blasenähnliche» Gebilde, wobei sich auf einer Seite des Einschlusses Mikrovilli befinden (Abb. 1b). Die Mikrovillus-Einschlüsse werden lysosomal abgebaut und die Autolysosomen tragen möglicherweise Mikrovillus-Fragmente in sich. Mikrovilli sind in vielen Fällen auf den basolateralen Membranen der Epithelzellen sichtbar.

Mikrovillus-Einschlüsse und eine Zunahme der Sekretgranula wurden sowohl im Dünn- als auch im Dickdarm, [18] nicht jedoch im Magen, beschrieben. Berichte, dass diese Merkmale im Nierenepithel oder in der Leber auftreten, liegen nicht vor, es gibt aber sehr wohl Berichte über ihr Auftreten im Epithel der Gallenblase.

Wie bereits durch den Begriff Mikrovillusatrophie angedeutet, liegt bei dieser Erkrankung eine Veränderung des Bürstensaums vor. Man muss sich jedoch bewusst machen, dass bei einer kongenitalen Mikrovillusatrophie die Mikrozotten der Kryptenregion normal erscheinen, während sie auf den Zotten entweder verkürzt sind oder ganz fehlen. Bei «late-onset»-Fällen sind die Mikrovilli im unteren Bereich der Zotten normal. Bei der «atypischen» Zottenatrophie sind die Mikrovilli auf den oberen Kryptenzellen verkürzt oder fehlen vollständig, treten dann aber wieder auf dem an der Oberfläche freiliegenden Epithel auf. Diese Muster der Mikrovillusatrophie verlaufen parallel zur Verteilung der Sekretgranula, d.h. bei Vorliegen von Sekretgranula verkürzen sich die Mikrovilli und bei Abnahme der Sekretgranula treten die Mikrovilli wieder auf [12].

\section{Diagnose}

Mittels PAS-Färbung und Transmissionselektronenmikroskopie (TEM) von aus dem proximalen Dünndarm entnommenen Schleimhautbiopsien lässt sich ohne weiteres eine Diagnose stellen. Das einfachste Verfahren besteht darin, bei gut ausgerichtetem formalinfixiertem Gewebe eine PAS-Färbung durchzuführen und anschlies- 
send im apikalen Zytoplasma des Epithels - in der Regel in der oberen Krypten- bzw. unteren Zottenregion - nach einer Ansammlung von PAS-positivem Material zu suchen. Wird eine solche Ansammlung entdeckt, sollte mittels TEM untersucht werden, ob Mikrovillus-Einschlüsse sichtbar sind. Da diese in der Regel in den Oberflächenepithelien vorliegen, ist die Ausrichtung der Gewebeprobe wichtig und bei einer quer geschnittenen Gewebeprobe werden sich Schwierigkeiten ergeben.

Als diagnostische Methoden wurden diverse Anfärbetechniken vorgeschlagen, u.a. AP (alkalische Phosphatase) [37], CEA (karzinoembryonales Antigen) [38] und CD10-Antigen [39], um die Notwendigkeit der Elektronenmikroskopie zu umgehen, die zwar wichtig ist [40], jedoch als technisch anspruchsvoll gilt. Mit diesen Methoden lassen sich die Mikrovillus-Einschlüsse besser nachweisen als mittels der bei PAS-Färbung beobachteten Veränderung. Wenn man beide auffälligen Befunde in Betracht zieht, d.h. den mittels Anfärbetechnik und den im Elektronenmikroskop erhaltenen Befund, so ist die Wahrscheinlichkeit einer endgültigen Diagnose höher; verlässt man sich hingegen lediglich auf die immunhistochemischen Untersuchungen, so besteht die Gefahr einer Fehldiagnose, da auch Autophagosomen eine positive Färbung aufweisen können.

\section{Pathogenese}

Die Pathogenese der Krankheit ist unbekannt. Das Vorliegen von Mikrovillus-Einschlüssen legt den Schluss nahe, dass möglicherweise eine grundlegende Störung im Aufbau des Zytoskeletts eine Rolle spielt. Die Tatsache, dass man bei kongenitalen und «late-onset»-Fällen in den frühen Stadien der Zellentwicklung normale Mikrovilli findet und dass die Mikrozotten bei den Fällen der «atypischen» Mikrovillusatrophie [12] wieder auftreten, lässt dies jedoch als unwahrscheinlich erscheinen. Die wichtigsten Zytoskelettproteine der Mikrovilli befinden sich im apikalen Zytoplasma der Kryptenzellen und stellen die für das Wachstum und den Erhalt des Bürstensaums notwendige Versorgung sicher [41]. Möglicherweise liegt eine Störung dieses Systems vor, die zu einer vorzeitigen Bildung der Mikrovilli in der Zelle (Abb. 1a) bzw. zu einem unangemessenen Mikrovillus-Wachstum auf der lateralen Zellmembran führt. Mikrovillus-Einschlüsse können durch Phagozytose der apikalen Membran entstehen (Abb. 1b) [4, 42]. Die Aufnahme von Material aus dem Darmlumen in die Mikrovillus-Einschlüsse bekräftigt diese Hypothese [42]. Diese Möglichkeiten sind jedoch nicht unbedingt ausschliesslicher Natur.
Die Prävalenz der Sekretgranula (Abb. 2) lässt auf einen Defekt in der Exozytose des für den Bürstensaum bestimmten Materials schliessen. Die konstitutiven direkten und indirekten sekretorischen Wege der Bürstensaumenzyme sind intakt [43] (obwohl die Enzymspiegel aufgrund von Zellschäden niedriger sind [12]) und deuten auf einen Defekt in einer nicht identifizierten zellulären Transportbahn. In einer frühen Abhandlung war von einem Myosinmangel im Bürstensaum die Rede [44], der eine Störung der Actin-Myosin-Wechselwirkungen, die für die Bereitstellung der membranassoziierten Substanzen an den Bürstensaum verantwortlich sind, vermuten liess [45]. Ameen und Salas [46] wiesen ferner in einem Einzelfallbericht auf einen Transportdefekt der apikalen Membran hin.

Die Beschaffenheit der Sekretgranula ist unbekannt. Normalerweise nehmen sie 2,5\% des Volumens apikaler Zellen ein, bei der Mikrovillusatrophie dagegen 40\% [46]. Jüngere Daten zeigen, dass in den Sekretgranula acetylierte Sialinsäure und Blutgruppenantigene vorliegen, was darauf hindeutet, dass die Sekretgranula einen glycocalyxartigen Stoff enthalten, der an der Exozytose gehindert wird [47]. Diese Vermutung wird durch den faserigflockigen Inhalt der Sekretgranula bestärkt (Abb. 1a). Die Glycocalyx bildet eine hydrophile polyanionische Gelhüllschicht auf der Zelloberfläche, welche die Oberflächenladung erhält, vor physischen Traumen schützt und den Zugang von Ionen und Makromolekülen reguliert [48]. Ein Glycocalyx-Mangel würde sich stark auf die normale Zellfunktion auswirken und die Ansammlung von Abfallstoffen in der Zelle möglicherweise eine Unterbrechung der normalen Zellprozesse zur Folge haben. In utero wird der Darm mit amniotischer Flüssigkeit gespült, die eine ähnliche Osmolalität aufweist wie das Blutserum, was zur Folge hat, dass sich im Darm kein osmotischer Druck aufbaut. Dies ändert sich nach der Geburt drastisch und das Fehlen einer funktionstüchtigen Glycocalyx im Darm könnte eine Erklärung bieten für das schnelle Einsetzen der Symptome und den Umstand, dass die meisten Fälle der Mikrovillusatrophie darauf beruhen, dass keine Exozytose der Glycocalyx stattfinden kann.

\section{Tufting-Enteropathie/Epithelzelldysplasie}

Reifen et al. [50] beschrieben die Tufting-Enteropathie bzw. Epithelzelldysplasie bei drei Kindern. Laut der Berichte setzte die Diarrhoe in der Neugeborenenphase ein. Ein früherer Fall wurde von Davidson et al. [4] be- 
schrieben und unterstreicht, dass die intraktable Diarrhoe in diesen Fällen auf unterschiedliche Ursachen zurückzuführen ist. In zwei Fällen konnte das Stuhlvolumen durch entsprechende Ernährung drastisch verringert und dank supportiver Ernährung eine normale Wachstumsrate erzielt werden. Die wichtigste Beobachtung war das Vorliegen einer Enteropathie mit Bündeln eng nebeneinanderliegender Oberflächenenterozyten und Rundung der apikalen Membran, die sich vom Epithel abzulösen schienen. Diese Zellbündel treten nicht nur bei dieser Erkrankung auf, sondern wurden auch bei Zöliakie, Mikrovillusatrophie, Autoimmun-Enteropathie sowie bei Kontrollpersonen gemeldet, wobei die Inzidenz in diesen Fällen jedoch sehr viel geringer war (90 vs. $61-<10 \%$ ) [50]. Im Kryptenepithel wurden diese Zellbündel nicht beobachtet. Es lagen keine charakteristischen ultrastrukturellen Merkmale vor, eine gesteigerte Zellinfiltration durch die Lamina propria war nicht festzustellen und die Enteropathie persistierte, obwohl sich der Zustand dank supportiver Ernährung in zwei Fällen verbesserte. Aufgrund dieser Eigenschaften wird diese Erkrankung in die Kategorie der kongenitalen Enterozytendefekte und nicht in die Kategorie der immunologischen Defekte eingeteilt. Es liegen Berichte über ähnliche Beobachtungen bei sechs weiteren Kindern vor [5], die zusätzliche Informationen über Veränderungen der Basalmembran enthalten, d.h. verringertes Laminin und erhöhtes Heparan-Sulfat. Die primäre und sekundäre Ursache der Veränderungen war jedoch nicht festzustellen. Patey et al. [52] untersuchten die Adhäsionsmoleküle und fanden eine Zunahme an Desmoglein, eine Veränderung der $\alpha_{2} \beta_{1}$-Integrin Verteilung entlang der Krypten-Zotten-Achse und eine Zunahme der Desmosomen. Sie kamen zu dem Schluss, dass Veränderungen der Zellen-Zellen bzw. der Zellen-Matrix-Interaktionen vorlagen und dass diese an der Pathogenese beteiligt waren.

Die Tufting-Enteropathie gilt im Allgemeinen als eine autosomal rezessive Erkrankung. Häufig liegt eine Konsanguinität der Eltern vor und die Krankheit tritt oft bei mehreren Geschwistern auf [15]. Reifen et al. [50] berichteten zwar, dass bei den von ihnen untersuchten Fällen keine Dickdarmveränderungen vorlagen, in weiteren Berichten wurde jedoch gezeigt, dass sich auch im Dickdarm einige Kryptenveränderungen (Verästelung und Pseudozysten) feststellen lassen [52]. Die Diagnose ist nicht unbedingt einfach, da sich die Prävalenz der Zellbündel im Laufe der Zeit ändert und zur Diagnosestellung bzw. zum definitiven Ausschluss einer Mikrovillusatrophie wiederholte Biopsien notwendig sind [15]. In manchen Fällen tritt möglicherweise eine begleitende
Entzündung der Lamina propria auf und durch Behandlung dieser Entzündung kann unter Umständen eine erhöhte Kalorienzufuhr erzielt werden [53]. Es sprechen einige Hinweise dafür, dass sich die Erkrankung im Laufe der Zeit legen könnte. Zwei erwachsene Patienten müssen inzwischen, nach dreizehn- bis fünfzehnjähriger Behandlung, nicht mehr parenteral ernährt werden [53] und bei einer Frau mit Tufting-Enteropathie wurde über eine erfolgreiche Schwangerschaft berichtet [54].

Weitere Klärung bezüglich dieser Erkrankung erwartet man sich von der molekulargenetischen Analyse. Es wurde ein Zusammenhang zwischen der Tufting-Enteropathie und Mehrfachmissbildung(en) mitgeteilt, [55] der jedoch möglicherweise eher zufällig als genetisch bedingt ist.

\section{Integrin-Mangel}

Lachaux et al. [56] berichteten vor kurzem über einen Säugling mit Pylorusatresie und intraktabler Diarrhoe. Im Lichtmikroskop zeigte sich eine umfassende starke Abschuppung der Epithelzellen vom Magen bis zum Rectum, bei der lediglich die Kryptenepithelien an Ort und Stelle blieben. Die elektronenmikroskopische Untersuchung des Darms ergab normale Desmosomen und eine Abtrennung der Lamina propria von der Basalmembran der Enterozyten. Man geht davon aus, dass diese Störung mit einem kongenitalen Mangel an $\alpha_{6} \beta_{4}$-Integrin verbunden ist, wobei es sich jedoch um eine intestinale Isoform des $\alpha_{6} \beta_{4}$-Integrins handeln muss, da eine normale kutane Expression von $\alpha_{6} \beta_{4}$-Integrin festgestellt wurde. Ein Defekt dieses Integrins liegt auch bei Epidermolysis bullosa vor, einer Erkrankung, die mit einer starken Abschuppung der Epidermis einhergeht. Wahrscheinlich liegt eine Mutation der intestinalen $\alpha_{6} \beta_{4}$-Isoform bzw. ein Mangel eines mit dieser Isoform verwandten intestinalen Integrins vor, das sich bei der immunhistochemischen Untersuchung als kreuzreaktiv erweist.

\section{Syndromatische Diarrhoe}

Bei einer kleinen Zahl von Patienten wurde die intraktable Diarrhoe als syndromatisch bedingt beschrieben $[57,58]$. Über ähnliche Fälle, die zum Tricho-HepatoEnterischen Syndrom gezählt wurden, wurde bereits früher berichtet [59, 61]. Patienten mit diesem Syndrom sind für ihr Gestationsalter zu klein und leiden unter einer in den ersten Lebensmonaten einsetzenden protra- 
hierten, therapieresistenten Diarrhoe, Lebererkrankung, Gesichtsdysmorphismus, geistiger Retardation und Haarveränderungen. Die Dünndarmmorphologie ist unterschiedlich und deckt das gesamte Spektrum von der schweren Zottenatrophie bis zur geringfügigen Verkürzung der Zotten ab. Auch eine Colitis wurde gemeldet [57]. Häufig liegt eine Konsanguinität der Eltern vor [58], und es gibt auch Berichte über Familien, in denen mehrere Geschwister unter diesem Syndrom leiden [59, 61], was auf eine autosomal rezessive Erkrankung schliessen lässt. Bei einigen Fällen lässt sich ein Immunmangel nachweisen, bei dem das Kind schlecht auf Impfungen anspricht und unter einer Hypogammaglobulinämie leidet.

Die Prognose scheint von Fall zu Fall unterschiedlich zu sein; einige Patienten sind an Infektionen oder Lebererkrankungen gestorben, andere müssen ganz oder teilweise parenteral ernährt werden und bei einem Fall ist eine orale Ernährung möglich [58]. Es wurde behauptet, die Verträglichkeit der enteralen Ernährung richte sich nach dem Zustand des Dünndarms [58]. Die Beziehung zwischen der syndromatisch bedingten intraktablen Diarrhoe und dem Tricho-Hepato-Enterischen Syndrom bedarf der näheren Untersuchung. Das klinische Muster lässt entweder auf Mutationen mehrerer, aufgrund eines Kopplungsungleichgewichts gemeinsam vererbter Gene oder auf eine Interferenz mit einer höheren Kontrollebene, beispielsweise einem Entwicklungsgen (PatterningGen), schliessen. Der Umstand, dass an der Haarentwicklung eine relativ geringe Zahl an Patterning-Genen beteiligt ist, ermöglicht unter Umständen eine gezieltere Suche der Kandidatenmutationen [15].

\section{Kongenitale Störungen der Glykosylierung und Proteinverlustenteropathie}

Die Diarrhoe ist mit verschiedenen kongenitalen Störungen der Glykolysierung verbunden und ein Bericht beschrieb einen zwei Wochen alten Säugling, bei dem sich als Symptom eine schwere intraktable Diarrhoe manifestierte, die bei parenteraler Ernährung persistierte und mit einer ausgeprägten Hypoalbuminämie einherging [62]. Der Patient entwickelte neurologische Symptome, sprach nicht auf eine Behandlung mit Mannose an und verstarb im Alter von 24 Monaten an Leberinsuffizienz. Die zugrunde liegende Störung der Glykosylierung konnte, abgesehen von einem Typ-1-Defekt, nicht identifiziert werden.
Drei ähnliche Fälle von intraktabler Diarrhoe und schwerer Proteinverlustenteropathie wurden berichtet [63]. Bei diesen Fällen erschien die Dünndarmschleimhaut in der histologischen Untersuchung normal, aber auf den lateralen Membranen der Epithelzellen fand sich kein Heparan-Sulfat. Der Zusammenhang zwischen Heparan-Sulfat, Proteinverlustenteropathie und Entzündung wurde vor kurzem in einem Experiment nachgewiesen [64]. Alle drei Patienten benötigten eine total parenterale Ernährung, bei der die Diarrhoe jedoch dennoch persistierte. Neurologische Veränderungen oder andere Symptome einer Störung der Glykolysierung wurden nicht beschrieben, aber es wurde ein Fall gemeldet, bei dem eine kongenitale Störung der Glykolysierung vom Typ 1 c vorlag, die mit einer schweren Hypoalbuminämie einherging und bei der sich auf dem Darmepithel ebenfalls kein Heparan-Sulfat fand [65]. Patienten mit einer kongenitalen Störung der Glykolysierung vom Typ-1b leiden ferner unter schwerer Diarrhoe und Proteinverlustenteropathie [66], so dass bei den unterschiedlichen Krankheiten durchaus Ähnlichkeiten vorhanden sein können.

\section{Sonstige Ätiologien und Zusammenfassung}

Obwohl die Fälle der intraktablen Diarrhoe intensiv erforscht wurden, gibt es nach wie vor viele Fälle, bei denen sich die Suche nach charakteristischen diagnostischen Merkmalen als äusserst schwierig erweist. Die Entdeckung der genetischen Grundlagen von Krankheiten, die mit charakteristischen Merkmalen einhergehen, wirft hoffentlich Licht auf Aspekte dieser idiopathischen Erkrankungen. Es ist zweifelsohne dem Engagement des in der pädiatrischen Gastroenterologie tätigen medizinischen Personals, das die von solchen Störungen der Enterozytenentwicklung betroffenen Kinder versorgt, zu verdanken, dass sich Behandlungswege und unterschiedliche Diagnosen herauskristallisiert haben, die eine gezielte und produktive Forschung ermöglichen. Trotz medizinischer Intervention jedoch persistieren viele Fällen von Durchfallerkrankungen und Enteropathien, die auf kongenitalen, durch Enterozytendefekte verursachten Krankheiten beruhen, bis eine Darmtransplantation vorgenommen wird. Die Heilung dieser komplexen und schwerwiegenden Erkrankungen wird sicher erst möglich sein, nachdem eine erfolgreiche Genbehandlung gefunden worden ist. 


\section{Literatur}

1 Avery GB, Villavicencio O, Lilly JR, Randolph JG: Intractable diarrhoea in early infancy. Pediatrics 1968;41:712-722.

-2 Larcher VF, Shepherd R, Francis DEM, Harries JT: Protracted diarrhoea in infancy. Analysis of 82 cases with particular reference to diagnosis and management. Arch Dis Child 1977;52:597-605

-3 Hyman CJ, Reiter J, Rodnan J, Drash AL: Parenteral and oral alimentation in the treatment of the nonspecific protracted diarrheal syndrome of infancy. J Pediatr 1971;78:17-29.

-4 Davidson GP, Cutz E, Hamilton JR, Gall DG: Familial enteropathy: a syndrome of protracted diarrhea from birth, failure to thrive, and hypoplastic villous atrophy. Gastroenterology 1978;75:783-790.

-5 Candy DCA, Larcher VF, Cameron DJS, et al: Lethal familial protracted diarrhoea. Arch Dis Child 1981;56:15-23.

-6 Cuenod B, Brousse N, Goulet O, et al: Classification of intractable diarrhea in infancy using clinical and immunohistological criteria. Gastroenterology 1990;99:1037-1043.

-7 Goulet OJ, Brousse N, Canioni D, WalkerSmith JA, Schmitz J, Phillips AD: Syndrome of intractable diarrhoea with persistent villous atrophy in early childhood: a clinicopathological survey of 47 cases. J Pediatr Gastroenterol Nutr 1998;26:151-161

-8 Goulet O, Ruemmele F, Lacaille F, Colomb V: Irreversible intestinal failure. J Pediatr Gastroenterol Nutr 2004;38:250-269.

-9 Sanderson IR, Risdon RA, Walker-Smith JA: Intractable ulcerating enterocolitis of infancy. Arch Dis Child 1991;66:295-299.

10 Thapar N, Shah N, Ramsay AD, Lindley KJ, Milla PJ: Long-term outcome of intractable ulcerating enterocolitis of infancy. J Pediatr Gastroenterol Nutr 2005;40:582-588.

-11 Cutz E, Rhoads JM, Drumm B, Sherman PM, Durie PR, Forstner GG: Microvillus inclusion disease: an inherited defect of brush-border assembly and differentiation. $\mathrm{N}$ Engl $\mathrm{J}$ Med 1989;320:646-651.

12 Phillips AD, Schmitz J: Familial microvillous atrophy: a clinicopathological survey of 23 cases. J Pediatr Gastroenterol Nutr 1992;14: 380-396.

13 Cutz E, Sherman PM, Davidson GP: Enteropathies associated with protracted diarrhea of infancy: clinicopathological features, cellular and molecular mechanisms. Pediatr Pathol Lab Med 1997; 17:335-368.

14 Sherman PM, Mitchell DJ, Cutz E: Neonatal enteropathies: defining the causes of protracted diarrhea of infancy. J Pediatr Gastroenterol Nutr 2004;38:16-26.

15 Goulet O, Phillips AD: Congenital enteropathy involving intestinal mucosa development; in Walker WA, Goulet O, Kleinman RE, Sanderson IR, Sherman P, Shneider B (eds): Paediatric Gastrointestinal Disease. Burlington, Decker, 2004, pp 922-931.
16 Schmitz J, Ginies JL, Arnaud-Battandier F, et al: Congenital microvillous atrophy: a rare cause of neonatal intractable diarrhoea. Pediatr Res 1982;16:1014.

17 Goutet JM, Boccon-Gibod L, Chatelet F, Ploussard JP, Navarro J, Polonovski Cl: Familial protracted diarrhoea with hypoplastic villous atrophy: report of two cases. Pediatr Res 1982;16:1045.

18 Phillips AD, Jenkins P, Raafat F, WalkerSmith JA: Congenital microvillous atrophy: specific diagnostic features. Arch Dis Child 1985;60:135-140.

19 Beck NS, Chang YS, Kang IS, Park WS, Lee HJ, Suh YL: Microvillus inclusion disease in two Korean infants. J Korean Med Sci 1997; 12:452-456.

20 Kaneko K, Shimizu T, Fujiwara S, Igarashi J, Ohtomo Y, Yamashiro Y: Microvillous inclusion disease in Japan. J Pediatr 1999;135: 400.

21 Pohl JF, Shub MD, Trevelline EE, et al: A cluster of microvillous inclusion disease in the $\mathrm{Na}-$ vajo population. J Pediatr 1999;134:103106.

22 Kennea N, Norbury R, Anderson G, Tekay A: Congenital microvillous inclusion disease presenting as antenatal bowel obstruction. Ultrasound Obstet Gynecol 2001;17:172-174.

23 Makela S, Kere J, Holmberg C, Hoglund P: SLC26A3 mutations in congenital chloride diarrhea. Hum Mutat 2002;20:425-438.

24 Keller KM, Wirth S, Baumann W, Sule D, Booth IW: Defective jejunal brush border membrane sodium/proton exchange in association with lethal familial protracted diarrhoea. Gut 1990;31:1156-1158.

25 Croft NM, Howatson AG, Ling SC, Nairn L, Evans TJ, Weaver LT: Microvillous inclusion disease: an evolving condition. J Pediatr Gastroenterol Nutr 2000;31:185-189.

26 Ruemmele FM, Jan D, Lacaille F, et al: New perspectives for children with microvillous inclusion disease: early small bowel transplantation. Transplantation 2004;77:1024-1028.

27 Randak C, Langnas AN, Kaufman SS, et al: Pretransplant management and small bowelliver transplantation in an infant with microvillus inclusion disease. J Pediatr Gastroenterol Nutr 1998;27:333-337.

28 Nathavitharana KA, Green NJ, Raafat F, Booth IW: Siblings with microvillous inclusion disease. Arch Dis Child 1994;71:71-73.

29 Assmann B, Hoffmann GF, Wagner L, et al: Dihydropyrimidinase deficiency and congenital microvillous atrophy: coincidence or genetic relation? J Inherit Metab Dis 1997;20:681688

30 Heinz-Erian P, Schmidt H, Le Merrer M, Phillips AD, Kiess W, Hadorn HB: Congenital microvillus atrophy in a girl with autosomal dominant hypochondroplasia. J Pediatr Gastroenterol Nutr 1999;28:203-205.
31 Raafat F, Green NJ, Nathavitharana KA, Booth IW: Intestinal microvillous dystrophy: a variant of microvillous inclusion disease or a new entity? Hum Pathol 1994;25:1243-1248.

32 Mierau GW, Wills EJ, Wyatt-Ashmead J, Hoffenberg EJ, Cutz E: Microvillous inclusion disease: report of a case with atypical features. Ultrastruct Pathol 2001;25:517-521.

33 Weeks DA, Zuppan CW, Malott RL, Mierau GW: Microvillous inclusion disease with abundant vermiform, electron-lucent vesicles. U1trastruct Pathol 2003;27:337-340.

-34 Walker-Smith JA, Phillips AD, Walford N, et al: Intravenous epidermal growth factor/urogastrone increases small intestinal cell proliferation in congenital microvillous atrophy. Lancet 1985;ii:1239-1240.

35 Groisman GM, Sabo E, Meir A, Polak-Charcon $\mathrm{S}$ : Enterocyte apoptosis and proliferation are increased in microvillous inclusion disease (familial microvillous atrophy). Hum Pathol 2000;31:1404-1410.

36 Phillips AD, Szafranski M, Man LY, Wall WJ: Periodic acid-Schiff staining abnormality in microvillous atrophy: photometric and ultrastructural studies. J Pediatr Gastroenterol Nutr 2000; 30:34-42.

37 Lake BD: Microvillus inclusion disease: specific diagnostic features shown by alkaline phosphatase histochemistry. J Clin Pathol 1988;41:880-882.

-38 Groisman GM, Ben-Izhak O, Schwersenz A, Berant M, Fyffe B: The value of polyclonal carcinoembryonic antigen immunostaining in the diagnosis of microvillus inclusion disease. Hum Pathol 1993;24:1232-1237.

-39 Groisman GM, Amar M, Livne E: CD10: a valuable tool for the light microscopic diagnosis of microvillous inclusion disease (familial microvillous atrophy). Am J Surg Pathol 2002; 26:902-907.

40 Bell SW, Kerner JA, Sibley RK: Microvillous inclusion disease. The importance of electron microscopy for diagnosis. Am J Surg Pathol 1991;15:1157-1164.

41 Fath KR, Obenauf SD, Burgess DR: Cytoskeletal protein and mRNA accumulation during brush border formation in adult chicken enterocytes. Development 1990;109:449-459.

42 Reinshagen K, Naim HY, Zimmer KP: Autophagocytosis of the apical membrane in microvillus inclusion disease. Gut 2002;51:514521.

43 Phillips AD, Fransen JAM, Hauri HP, Sterch E: The constitutive exocytotic pathway in microvillous atrophy. J Pediatr Gastroenterol Nutr 1993; 17:239-246.

44 Carruthers L, Phillips AD, Dourmashkin R, Walker-Smith JA: Biochemical abnormality in brush border membrane protein of a patient with congenital microvillous atrophy. J Pediatr Gastroenterol Nutr 1985;4:902-907.

-45 Fath KR, Trimbur GM, Burgess DR: Molecular motors are differentially distributed on Golgi membranes from polarized epithelial cells. J Cell Biol 1994;126:661-675. 
-46 Ameen NA, Salas PJ: Microvillus inclusion disease: a genetic defect affecting apical membrane protein traffic in intestinal epithelium. Traffic 2000; 1:76-83.

47 Phillips AD, Brown A, Hicks S, et al: Acetylated sialic acid residues and blood group antigens localise within the epithelium in microvillous atrophy indicating internal accumulation of the glycocalyx. Gut 2004;53:1764-1771.

-48 Gupta BL: The relationship of mucoid substances and ion and water transport, with new data on intestinal goblet cells and a model for gastric secretion. Symp Soc Exp Biol 1989;43: 81-110.

49 Gillibrand PN: Changes in the electrolytes, urea and osmolality of the amniotic fluid with advancing pregnancy. J Obstet Gynaecol $\mathrm{Br}$ Commonw 1969;76:898-905.

>50 Reifen RM, Cutz E, Griffiths AM, Ngan BY, Sherman PM: Tufting enteropathy: a newly recognized clinicopathological entity associated with refractory diarrhea in infants. J Pediatr Gastroenterol Nutr 1994;18:379-385.

$\checkmark 51$ Goulet O, Kedinger M, Brousse N, et al: Intractable diarrhea of infancy with epithelial and basement membrane abnormalities. J Pediatr 1995; 127:212-219.

52 Patey N, Scoazec JY, Cuenod-Jabri B, et al: Distribution of cell adhesion molecules in infants with intestinal epithelial dysplasia (tufting enteropathy). Gastroenterology 1997;113: 833-843.
53 Schaeppi M, Shah NF, Long S, et al: Tufting enteropathy also affects the colon. J Pediatr Gastroenterol Nutr 1999;28:569.

54 Cameron DJ, Barnes GL: Successful pregnancy outcome in tufting enteropathy. J Pediatr Gastroenterol Nutr 2003;36:158.

55 Abely M, Hankard GF, Hugot JP, Cezard JP, Peuchmaur M, Navarro J: Intractable infant diarrhea with epithelial dysplasia associated with polymalformation. J Pediatr Gastroenterol Nutr 1998;27:348-352.

56 Lachaux A, Bouvier R, Loras-Duclaux I, Chappuis JP, Meneguzzi G, Ortonne JP: Isolated deficient alpha6beta4 integrin expression in the gut associated with intractable diarrhea. J Pediatr Gastroenterol Nutr 1999;29:395_ 401.

57 Girault D, Goulet O, Le Deist F, et al: Intractable infant diarrhea associated with phenotypic abnormalities and immunodeficiency. J Pediatr 1994;125:36-42.

58 Martinez-Vinson C, Goulet O, Berrebi D, et al: Syndromatic diarrhea in children. Report of 8 cases. J Pediatr Gastroenterol Nutr 2005;40: 651.

59 Verloes A, Lombet J, Lambert Y, et al: Trichohepato-enteric syndrome: further delineation of a distinct syndrome with neonatal hemochromatosis phenotype, intractable diarrhea, and hair anomalies. Am J Med Genet 1997;68: 391-395.

60 Landers MC, Schroeder TM: Intractable diarrhea of infancy with facial dysmorphism, trichorrhexis nodosa, and cirrhosis. Pediatr Dermatol 2003;20:432-435.
61 Stankler L, Lloyd D, Pollitt RJ, Gray ES, Thom H, Russell G: Unexplained diarrhoea and failure to thrive in 2 siblings with unusual facies and abnormal scalp hair shafts: a new syndrome. Arch Dis Child 1982;57:212-216.

-62 Mention K, Michaud L, Dobbelaere D, Guimber D, Gottrand F, Turck D: Neonatal severe intractable diarrhoea as the presenting manifestation of an unclassified congenital disorder of glycosylation (CDG-x). Arch Dis Child Fetal Neonatal Ed 2001;85:F217-F219.

63 Murch SH, Winyard PJ, Koletzko S, et al: Congenital enterocyte heparan sulphate deficiency with massive albumin loss, secretory diarrhoea, and malnutrition. Lancet 1996;347: 1299-1301.

64 Bode L, Eklund EA, Murch S, Freeze HH: Heparan sulfate depletion amplifies TNF-alpha-induced protein leakage in an in vitro model of protein-losing enteropathy. Am J Physiol Gastrointest Liver Physiol 2005;288: G1015-G1023

65 Westphal V, Murch S, Kim S, et al: Reduced heparan sulfate accumulation in enterocytes contributes to protein-losing enteropathy in a congenital disorder of glycosylation. Am J Pathol 2000;157:1917-1925.

66 Niehues R, Hasilik M, Alton G, et al: Carbohydrate-deficient glycoprotein syndrome type Ib. Phosphomannose isomerase deficiency and mannose therapy. J Clin Invest 1998;101: 1414-1420. 